\title{
Transient Global Amnesia (TGA) as First Presenting Sign of Neurodegenerative Disorders
}

\author{
Cotelli Maria Sofia ${ }^{1}$, Manelli Filippo ${ }^{2}$, Tosana Elisa ${ }^{1}$, Turla Marinella ${ }^{1}$ \\ ${ }^{1}$ Neurology Unit, Azienda Socio Sanitaria Territoriale Valcamonica (Esine), Brescia, Italy \\ ${ }^{2}$ Department of Emergency and Urgency, Azienda Socio Sanitaria Territoriale Valcamonica (Esine), Brescia, Italy \\ Email address: \\ cotellim@gmail.com (C. M. Sofia), filippo.manelli@gmail.com (M. Filippo), elisatosana@gmail.com (T. Elisa), \\ marinellaturla@gmail.com (T. Marinella) \\ ${ }^{*}$ Corresponding author
}

\section{To cite this article:}

Cotelli Maria Sofia, Manelli Filippo, Tosana Elisa, Turla Marinella. Transient Global Amnesia (TGA) as First Presenting Sign of Neurodegenerative Disorders. Clinical Medicine Research. Special Issue: Rare and Degenerative Disorders in Neurology.

Vol. 8, No. 5, 2019, pp. 115-118. doi: 10.11648/j.cmr.20190805.14

Received: September 15, 2019; Accepted: October 8, 2019; Published: October 17, 2019

\begin{abstract}
Transient global amnesia (TGA) is a clinical syndrome characterized by the sudden onset of anterograde amnesia, accompanied by repetitive questioning, sometimes with a retrograde component, lasting up to 24 hours, without compromise of other neurologic functions. Although various factors, such as migraine, focal ischemia, venous flow abnormalities, and epileptic phenomena, can be considered as involved in the pathophysiology and differential diagnosis of TGA, the factors triggering the emergence of these lesions are still elusive. Recent data suggest that the vulnerability of CA1 neurons to metabolic stress plays a pivotal part in the pathophysiological cascade, leading to an impairment of hippocampal function during TGA. We report three cases on neurodegenerative disorders presenting with transitory global amnesia, all evaluated at the department of emergency of Esine Hospital (Brescia), who developed all neurodegenerative disorders. It is important, when performing neurological evaluation in the department of emergency, to consider the possible presence of underlying cognitive impairment or behavioral symptoms especially in older people. Neurological evaluation should take into consideration the presence of piramidal, extrapiramidal or frontal sign release positivity or the presence of higher cortical functions impairment. It should be important to refer these patients to appropriate neurological evaluation in appropriate Centers for Neurodegenerative Disorders.
\end{abstract}

Keywords: Amnesia, Neurodegenerative, Emergency

\section{Introduction}

Amnesia, or memory loss, often is an early hallmark of mild cognitive impairment or dementia. Because the memory problems evolve gradually, initial symptoms may be subtle, and the patient and family often attribute such memory problems to simple aging [1]. As the memory loss worsens, sometimes a particular upsetting event "unmasks" the problem. In high-functioning persons who can compensate well for mild memory losses, it is possible to attribute the onset of memory loss to a perceived precipitating event, such as an episode of transient global amnesia (TGA); however, the event simply may represent the first time that the gradually evolving problem comes to attention [1]. We report three cases on neurodegenerative disorders presenting with transitory global amnesia, all evaluated at the department of emergency of Esine Hospital (Brescia).

\subsection{Case 1}

A 67 years-old caucasian woman was admitted twice to the department of emergency due to two episodes of transitory global amnesia, apparently improving in about 24 hours. Her medical history was unremarkable except for hypertension and also her familial history was unremarkable. Neurological examination showed mild hypomimia with bilateral ophtalmoparesis and bradikynesia. Brain computer tomography resulted normal and blood exams were all normal. She was discharged with prescription of 
neuropsychological assessment of cognitive and behavioral functions using a set of standardized tests that performed after about six months, which revealed impaired executive functions (attentive matrices test, Trail- Making Test A and $\mathrm{B}$, phonemic and semantic fluency, frontal assessment battery were all altered). Her daughter referred that, after these episodes, she started to become "apathic, with important social isolation, hyperphagia with preference for sweet foods with consequent weight gain of about $11 \mathrm{Kg}$ in a few months". Magnetic resonance revealed bilateral signal alterations at gradient echo sequences (cerebral amyloid angiopathy?) together with cortico-subcortical atrophy (figure 1) and white matter leukoencephalopathy. Diagnosis of frontotemporal behavioral variant was performed after genetic and lumbar puncture exams. Neurological examination and behavioral symptoms quickly worsened (she developed extrapyramidal syndrome, global aphasia urge incontinence, lack of inhibition, loss of empathy, gambling) she and was consequently in-hospitalized in a retirement home within two years.

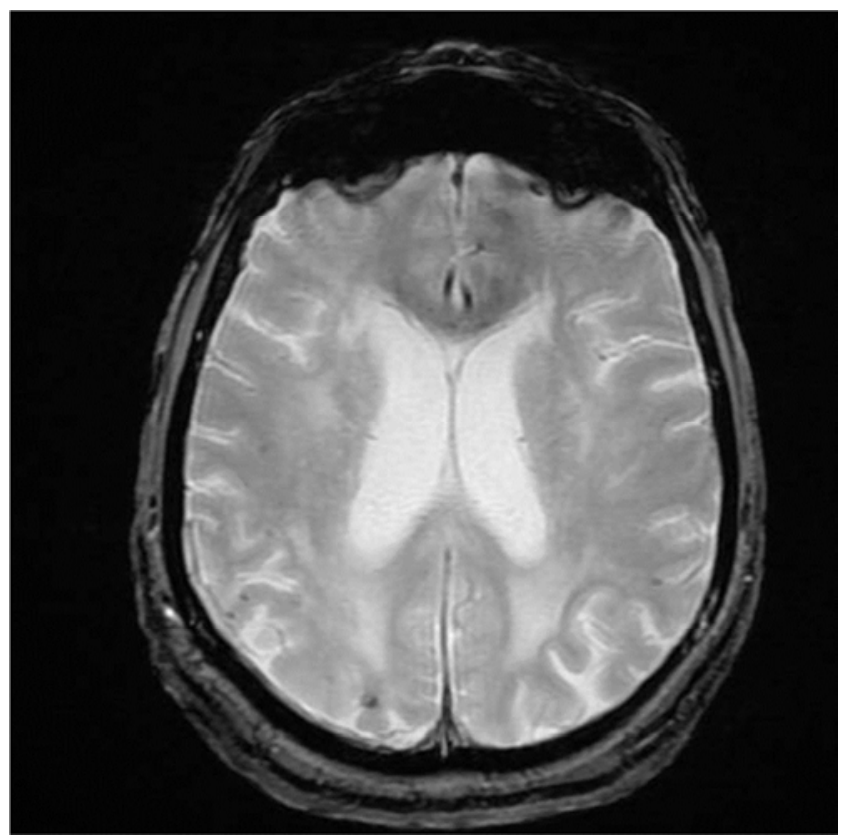

Figure 1. Gradient echo axial sequence.

\subsection{Case 2}

A 74 years-old patient was admitted to general emergency with referred sudden episode of transitory global amnesia, after which he resulted unable to selectively recognize his wife. Brain computer tomography resulted normal, blood exams were all normal. He suffered from hypertensive cardiopathy. His neurological examination was normal except for mild hypomimia; he appeared oriented in space and time but upset and distressed, so benzodiazepines were orally administered with apparent improvement, and he was discharged. Due to the persistence of visual agnosia in the following days he performed brain magnetic nucleic resonance, which resulted compatible with cerebral amyloid angiopathy (multiple bilateral emispheric lesions with loss of signal at T2*-weighted gradient-echo- GRE) (figure 2). Electroencephalography revealed brain global slow theta activity. During the following months his neurological examination didn't change while his visual agnosia worsened (he was unable to recognize even his two daughters). He also developed allucinations and compulsive behaviors. He was in-hospitalized to psychiatry department due to persistent aggressiveness.

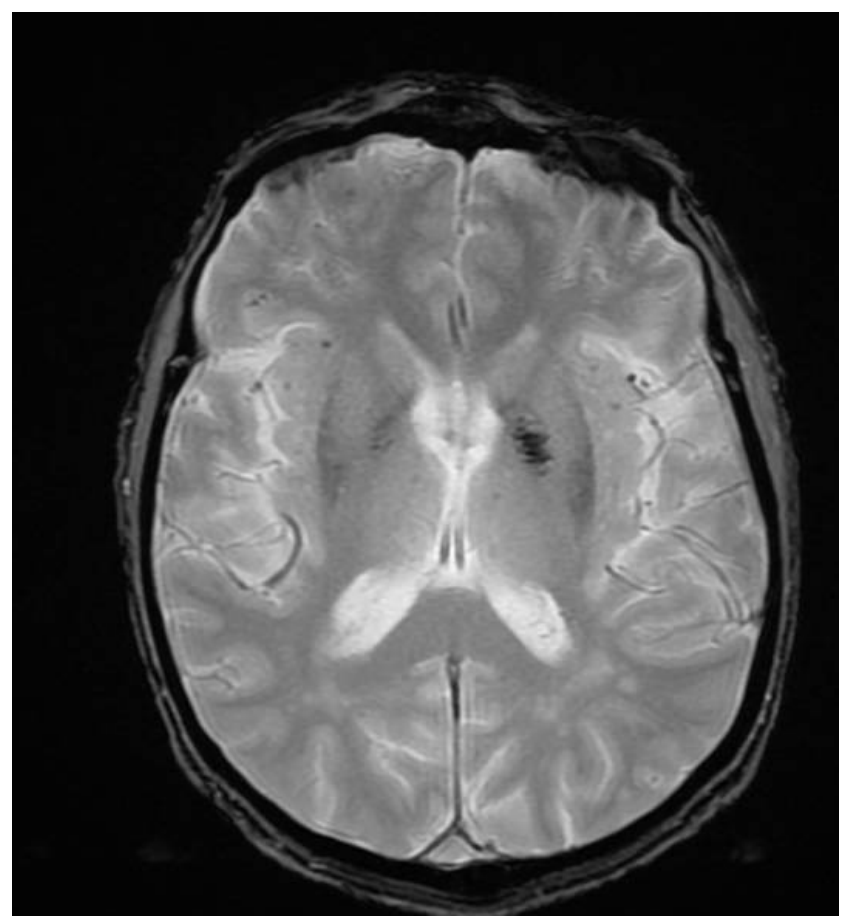

Figure 2. Gradient echo axial sequence.

\subsection{Case Report 3}

A 62 years-old woman was admitted to the department of emergency due to transient global amnesia. Brain computer tomography was unremarkable, blood exams were all normal, her medical history was unremarkable except for hypertension, even in her relatives defined her as "apathic with recent onset of hyperphagia". Her familial history was unremarkable but her parents were second cousins. She was discharged with indication to perform brain magnetic resonance, which showed diffuse cortical and subcortical atrophy with preminent parietal involvement, multiple fronto-parietal lacunar strokes (figure 3), for which acetylsalicylic acid was promptly started. She also performed neuropsychological assessment showing impairment of shortterm memory (mini-mental state examination- MMSE: 27/30 with pathological performances at both Trail making test $\mathrm{A}$ and B). Neurological examination resulted normal except for hyposmia, frontal release sign positivity (glabellar, snout reflex, bilateral palmomental reflex), bucco-facial apraxia. Diagnosis of neurodegenerative disorder- (frontotemporal degeneration versus corticobasal syndrome) was performed (after lumbar puncture, blood exams, brain tomoscintigraphy and repetition of neuropsychological screening). 


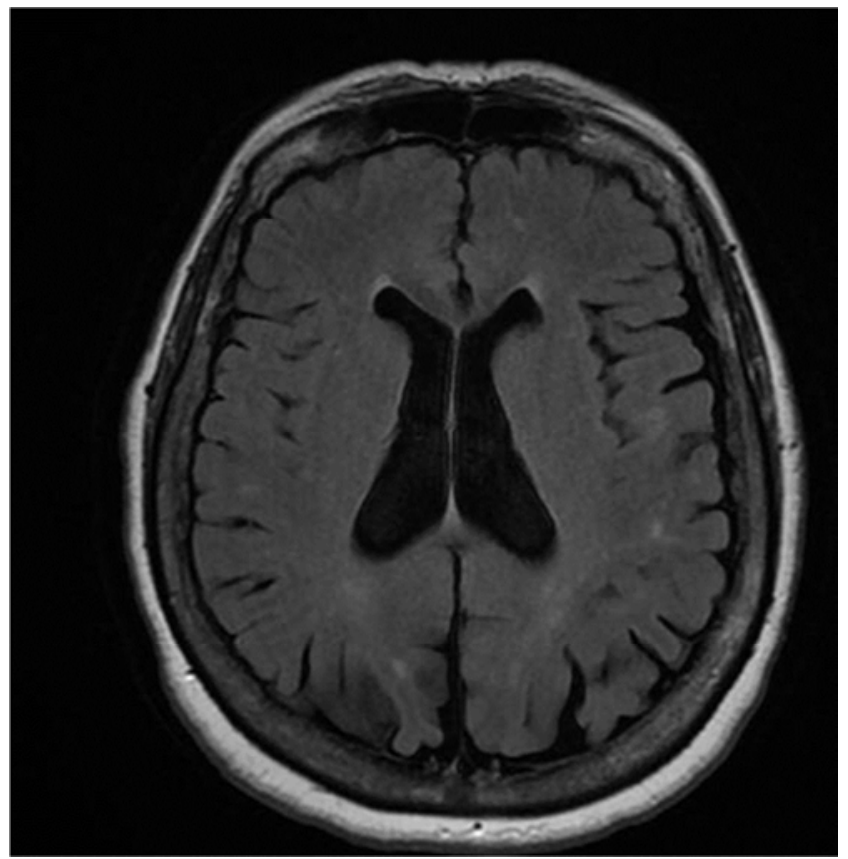

Figure 3. T2 FLAIR axial sequence.

\section{Discussion}

Transient global amnesia (TGA) is a clinical syndrome characterized by the sudden onset of an extraordinarily large reduction of anterograde and a somewhat milder reduction of retrograde episodic long-term memory [2]. Additionally, executive functions are described as diminished [2]. It was in 1956 that Guyotat and Courjon first described TGA [3]. In their study, they took a particular interest in describing the epidemiological characteristics of 16 patients (age, context of occurrence, duration, cognitive disorders, medical history and medical examination) [3].

Most systematic epidemiological studies show that the incidence of TGA ranges between 3 and 8 per 100000 people per year [4]. 29-32.75\% of attacks occur in people aged between 50 and 70 years, and occurrence in patients younger than 40 years of age is rare [4]. The rate of recurrence of a second or a third episode varies across studies; a recent thorough meta- analysis described the rate of annual recurrence to be between $6 \%$ and $10 \%$ [4]. Several small-scale studies show a slight female predominance [5].

The nature of precipitating events directly before a TGA has been widely studied. Events frequently described include sudden immersion in cold or hot water, physical exertion, emotional or psychological stress, pain, medical procedures, sexual intercourse, and Valsalva-associated maneuvers, and such events have been observed in $50-90 \%$ of documented attacks $[6,7]$. Several theories have been proposed regarding the etiology of TGA, but the underlying causes remain obscure [8]. Proposed risk factors include vascular risk factors, patent foramen ovale, and retrograde jugular venous flow [8], but also ischemia, migraine, seizures, psychological disorders particularly intense emotional stress $[9,10]$. A recent study found the role of these factors to be minimal and implicated cerebral venous hypertension caused by incompetence of the internal jugular valve [8]. Metabolic studies of the brain (cerebral positron emission tomography [PET] imaging with 18F-labeled fluorodeoxyglucose) showed that some patients had low metabolism in local areas related to memory [11]. Based on a study of 14 patients examined 3 or 4 days after the end of an episode of TGA, Kessler and colleagues proposed a major role for stress in the etiology of TGA and in the continuation of cognitive impairment [12]. Hodges and Warlow identified a putative precipitating event in $13 \%$ of their 63 patients with prospectively ascertained TGA or an emotionally stressful event of at least moderate severity during the 24 hours leading up to the attack in $14 \%$; in an additional $17 \%$, the attacks occurred in association with moderate to severe physical exertion [10] The diagnosis of TGA is primarily a clinical one and can be made if the following diagnostic criteria by Caplan and Hodges are fulfilled: (i) presence of an anterograde amnesia that is witnessed by an observer, (ii) no cloudig of consciousness or loss of personal identity, (iii) cognitive impairment limited to amnesia, (iv) no focal neurological or epileptic signs, (v) no recent history of head trauma or seizures, and (vi) resolution of symptoms within 24 h. A temporally graded retrograde amnesia is consistently present during the acute attack in patients with acute TGA, although this feature is not included in the present diagnostic criteria. Mild vegetative symptom such as headache, nausea, and dizziness might be present during the acute phase $[3,13]$. By definition, TGA is a self-limited condition that resolves without intervention; thus, there is no specific treatment indicated [14]. Although the mean time course of an amnestic episode in TGA is $4-6$ hours, with most resolving by 8 hours, proposed treatments could depend on uncovering the underlying etiology [15].

\section{Conclusions}

We reported three cases of dementia in all of which the first manifestation was transient global amnesia (TGA). They all were sent to the department of emergency and, after initial discharge, to neuropsychological ambulatory evaluation, often with prescription of neuroimaging exams. It is important, when performing neurological evaluation in the department of emergency, to consider the possible presence of underlying cognitive impairment or behavioral symptoms especially in older people. Neurological evaluation should take into consideration the presence of piramidal, extrapiramidal or frontal sign release positivity or the presence of higher cortical functions impairment when possible. It is also important to assess the effective cognitive improvement after AGT (the persistence of spatial or temporal confusion should be taken into consideration), and eventually consider other brain imaging exams (a brain CT performed in the emergency sometimes sometimes does not consider the presence of brain atrophy) such as MRI or tomoscintigraphy and a detailed neuropsychological assessment. 


\section{References}

[1] Daniel ES Early Diagnosis and Treatment of Dementia Presenting as Transient Global Amnesia in a 76-Year-Old Man. Prim Care Companion J Clin Psychiatry. 2004; 6 (6): 248-251.

[2] Spiegel DR, Smith J, Wade RR, Cherukuru N, Ursani A, Dobruskina Y, Crist T, Busch RF, Dhanani RM, Dreyer N. Transient global amnesia: current perspectives. Neuropsychiatr Dis Treat. 2017 Oct 24; 13: 2691-2703.

[3] Courjon J, Guyotat J. [Amnesic strokes] J Med Lyon. 1956; 37: 697-701.

[4] Bartsch T, Deuschl G. Transient global amnesia: functional anatomy and clinical implications. Lancet Neurol. 2010 Feb; 9 (2): 205-14.

[5] Arena JE, Brown RD, Mandrekar J et al. Long-term outcome in patients with transient global amnesia: a population-based study. Mayo Clin Proc. 2017; 92 (3): 399-405.

[6] Bartsch T, Alfke K, Stingele R, et al. Selective affection of hippocampal CA-1 neurons in patients with transient global amnesia without long-term sequelae. Brain 2006; 129: 287484.

[7] Lewis SL. A etiology of transient global amnesia. Lancet 1998; 352: 397-99.

[8] Maalikjy Akkawi N, Agosti C, Anzola GP, Borroni B, Magoni M, Pezzini A, Rozzini L, Vignolo LA, Padovani A. Transient global amnesia: a clinical and sonographic study. Eur Neurol. 2003; 49 (2): 67-71.

[9] Della Marca G, Broccolini A, Vollono C, et al. The stolen memory: a case of transient global amnesia. Biol Psychiatry. 2009; 67: e31-2. [PubMed] [Google Scholar].

[10] Marinella MA. Transient global amnesia and a father's worst nightmare. N Engl J Med. 2004; 350: 843-4.

[11] Jia J, Wang L, Yin L, Tang H. Contrast study on cognitive function with MRI and positron emission tomography imaging in transient global amnesia. Chin Med J (Engl). 2002 Sep; 115 (9): 1321-3.

[12] Kessler J, Markowitsch HJ, Rudolf J, Heiss WD. Continuing cognitive impairment after isolated transient global amnesia. Int J Neurosci. 2001; 106 (3-4): 159-68.

[13] Hodges JR, Warlow CP. Syndromes of transient amnesia: towards a classification. A study of 153 cases. J Neurol Neurosurg Psychiatry. 1990 Oct; 53 (10): 834-43.

[14] Brown J. Evaluation of transient global amnesia. Ann Emerg Med. 1997; 30 (4): 522-526.

[15] Quinette P, Guillery-Girard B, Dayan J, et al. What does transient global amnesia really mean? Review of the literature and thorough study of 142 cases. Brain. 2006; 129 (pt 7): $1640-1658$ 